\title{
Quantitative Analysis on Quality of Service Support using OTSC-DRX Mechanism in Long Term Evolution Device
}

\author{
R. Vassoudevan* and P. Samundiswary \\ Department of Electronics Engineering, School of Engineering and Technology, Pondicherry University, \\ Kalapet-605014, Puducherry, India; vassoudevan@rediffmail.com, samundiswarypu@gmail.com
}

\begin{abstract}
Objectives: Fourth Generation network namely Long Term Evolution (LTE) plays a vital role in the high data rate wireless communication systems with the support of enhanced Quality of Service (QoS). However the maintenance of QoS during the transmission in LTE network highly deteriorates the endurance of User Equipment (UE) battery. Therefore, it is required to mitigate the trade-off between QoS and power savings of the battery in the LTE network which is a big challenging task. Methods: In this paper, an attempt has been made to develop a new approach namely On-Timer to Short Cycle (OTSC) ratio based Discontinuous reception (DRX) mechanism for LTE network to minimize the packet loss ratio with optimal power consumption. This approach also extends the battery power endurance during the execution of high data rate applications. Further, the performance analysis is made on packet loss ratios with an influence of delay bound violated traffic by incorporating OTSC ratio in DRX mechanism. The packet loss ratio analyses are made with various OTSC ratios for different DRX parameters. The complete analyses are done using MATLAB simulator. Findings: The simulation results portray that the packet loss ratio is reduced based on the selection of appropriate OTSC ratio, during the occurrence of delay bound violation in the traffic. The simulation results also depict that $25 \%$ of improvement in the packet loss reduction is achieved with optimal power savings compared to that of the regenerative cycle techniques. Moreover the new approach provides an insight on the proper selection of packet buffer, short cycle timer and inactivity timer values in order to improve QoS and also extend the battery endurance. Applications: This method provides an easier approach for selecting the DRX parameters to improve QoS along with better battery power endurance.
\end{abstract}

Keywords: Discontinuous Reception, Guaranteed Bit Rate, Long Term Evolution, Packet Loss Ratio, Quality of Service

\section{Introduction}

Evolution of advanced physical layer techniques has made a remarkable revolution in the Fourth Generation Long Term Evolution (4G LTE).The high data rate transmission with lower latency in LTE transmission/ reception has made it to be a superior network quality compared to that of the Second and Third Generation (2G 3G) wireless communication systems. The lower latency of $1 \mathrm{~ms}$ transmission offered by LTE supports the high data rate services like online games, video conferencing, video streaming and voice over internet with enhanced QoS in the network. This attracts more and more smart phone users to add on to the network. The smart phone as a User Equipment (UE) in an LTE network plays a vital role to provide a nexus between the user and the outer world. The sophisticated in-built algorithms in UE provide flawless communication to cater the required data services for the user. However, the UE has to perform complex computations by using algorithms to handle high data rate and to process it for its

${ }^{*}$ Author for correspondence 
associated applications. Hence, the applications that handle high data rate make the UE to deplete the battery power more quickly compared to that of voice communication. Therefore the power savings in battery poses a challenging task in the UE of LTE.

The power consumption of LTE UE is 23 times more than that of Wi-Fi network and 15 times higher compared to that of the $3 \mathrm{G}$ technology ${ }^{1}$. The LTE specification release 8 proposes a mechanism namely Discontinuous Reception (DRX) mechanism ${ }^{2}$ to save power. The incorporation of this mechanism in UE ensures power savings by switching off its radio circuits when no data packets for transmission/reception are available. Thereby the UE enters into sleep mode. In every Transmission Time Interval (TTI), the DRX mechanism allows the UE to wake up and check the Physical Downlink Channel (PDCCH) for information regarding the arrival of any data packets intended for it. On sensing the data packets intended for it, UE enters into wake up period and switch on its radio circuits. The inactivity timer in UE is switched on for further sensing of data. Before expiry of inactivity timer, if any data packets are sensed, UE extends its wake-up period and the same process continues. Whereas if no data packets are sensed till the expiry of inactivity timer, UE enters into sleep mode to save the battery power. The process of switching the radio circuits on and off for transmission and reception of data for a defined period is organized by DRX timing parameters. Though the DRX mechanism provides power savings, an improper configuration of DRX parameters have worsen the battery performance. This may lead to the higher delay in transmission/reception of data between two consecutive on period. This deteriorates the QoS of high data rate applications. Hence, larger delay ensures higher power saving with poor QoS. Therefore the optimized DRX parameter is selected for reducing the power consumption and improving the QoS that poses further challenging task.

Various performance parameters on LTE network with Poisson data arrival model and ETSI traffic model are analyzed using direct implementation of DRX parameters ${ }^{3-5}$. Another study on UE power savings shows that a closely related DRX values show an improvement on the impacts of power saving trade off with delay constraints ${ }^{6}$. General performance study of power consumption with a web based application background is analyzed on the careful assignment of DRX parameters ${ }^{7}$. Assigning the shorter inactivity timer8 and larger wake-up delay ${ }^{9}$ while enabling DRX mechanism shows distinct power savings but the limits on applying the values have not specified. The performance analyses shows that an improper selection of the longer DRX cycle parameter in DRX configuration exhibits the higher power consumption with respect to the delay ${ }^{10}$. Impact on power savings is analyzed by a new concept of adaptively scheduling the sleep period depending on the network load using a Load Based Power Saving (LBPS) algorithm ${ }^{11}$. The performance on extended LBPS model shows $11 \%$ improvement in the power savings while integrating the network and user side. However, the method of configuring the parameters have not mentioned clearly in the work ${ }^{12}$. A study with revised LBPS schemes ${ }^{13}$ illustrates that the marginal improvement of $6 \%$ power savings is achieved while varying the input load of a network capacity. In the aspect of QoS, the performance of DRX is analyzed by considering the delay bound violated traffic ${ }^{14}$. The literature shows the direct method of applying the DRX parameters on minimizing the packet loss ratio. The study on packet delay variations and average frame delay ${ }^{15}$ is made based on the scheduling methods. However the power saving in relation to the delay has been not depicted in the previous work. Hence, an attempt is made in this paper incorporating the OTSC ratio method for packet loss ratio with optimal power saving aspect.

The section 2 describes the DRX mechanism and its importance in the LTE. The proposed QoS support technique and its analyses to reduce the packet loss ratio are dealt in Section 3 and Section 4 respectively. The section 5 presents an elaborate discussion on the simulation results. Finally the section 6 concludes briefly with the summary of work and conclusion.

\section{Long Term Evolution DRX Mechanism}

The Universal Mobile Telecommunication Systems (UMTS) introduced the Discontinuous Reception Mechanism (DRX) as a part of its specification for power savings in the Mobile Station (MS). In general, the UMTS DRX mechanism provides a simple on and off switching action. Though this technique exhibits an easy way of configuring, the disadvantage associated in this system is to mandatorily empty the base station buf- 
fers before it executes the DRX mechanism. This made the MS to wait before initiating the DRX till it receives all data from the base station. This technique made UMTS DRX mechanism to be less efficient.

To overcome this, Third Generation Partnership Project (3GPP) has released an extended version of specification on power saving techniques for the UE. The new version of enhanced techniques consists of two modes namely active mode and sleep mode durations through which LTE DRX further tunes the UE to achieve efficient power savings. Moreover, the data in the eNB buffers need not be emptied while initiating the DRX mechanism which has become an added advantage. This facility makes the UE to hibernate immediately irrespective of the availability of data in the buffer. The facility to configure in the light and deep sleep mode provides an opportunity for more power savings in UE.

Figure 1 represents the basic time line sequence of DRX mechanism of LTE. The figure also illustrates the active mode and sleep mode durations along with its associated DRX parameters. On executing the DRX mechanism, UE switches the radio circuit to on and off as per the configured values of DRX parameters. The selection of optimal values for DRX parameters acts as a governing factor for the power saving in UE. When no data for reception is identified, the UE enters into sleep mode from the active mode. However, the UE wakes up at regular timer period to sense any data packets intended for it. Since the DRX parameters are the prime focus on efficient power savings, it is necessary to understand each parameter. There are five major parameters which involve in power savings are governed by its corresponding timers. The working operations of all the timers are briefed as below and the Table 1 illustrates the DRX parameters and their ranges of values to yield higher power savings.

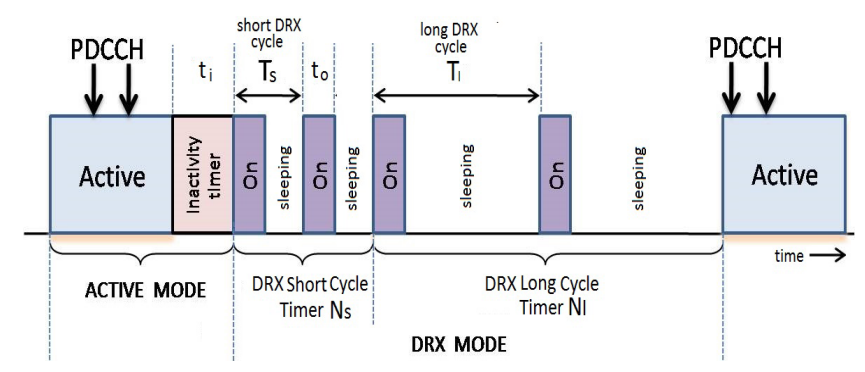

Figure 1. LTE DRX time line sequence.
Inactivity timer $t_{i}$ : This timer is initialized by the target UE on detection of PDCCH packet in the channel intended for it.

On Timer $t_{0}$ : On initiating this timer, UE wakes up for specified duration to detect the packets in PDCCH channel.

Short DRX Cycle $T_{s}$ : The UE enters into short sleep duration when no data reception is identified.

Long DRX Cycle $T_{i}$ : This timer makes the UE to enter into deep sleep mode after the expiry of short sleep duration $T_{s}$.

DRX Short Cycle Timer $N_{s}$ : This timer defines the number of consecutive short DRX cycle duration to be executed during light sleep mode.

Table 1. LTE standard DRX parameters

\begin{tabular}{|l|l|}
\hline DRX Parameter & Standard Value $(\mathrm{ms})$ \\
\hline Short DRX cycle $\mathrm{T}_{\mathrm{s}}$ & $\begin{array}{l}2,5,8,10,16,20,32,40,64,80,128,160, \\
256,320,512,640\end{array}$ \\
\hline Long DRX cycle $\mathrm{T}_{1}$ & $\begin{array}{l}10,20,32,40,64,80,128,160,256,320, \\
512,640,1024,1280,2048,2560\end{array}$ \\
\hline On duration $\mathrm{t}_{\mathrm{o}}$ & $1,2,3,4,5,6,8,10,20,30,40,50,60,80$, \\
& 100,200 \\
\hline Short Cycle Timer $\mathrm{N}_{\mathrm{s}}$ & $1,2,3,4,5,6,7,8,9,10,11,12,13,14,1$ \\
& 5,16 \\
\hline Inactivity Timer $\mathrm{t}_{\mathrm{i}}$ & $0,1,2,3,4,5,6,8,10,20,30,40,50,60$, \\
& $80,100,200,300,500,750,1280,192$ \\
& 0,2560 \\
\hline
\end{tabular}

Initially the UE starts listening for the arrival of $\mathrm{PDCCH}$ packets with the configured values of DRX parameters. On sensing the PDCCH packets, UE starts the inactivity timer $t_{i}$ and extends the on duration of radio circuits to detect the further arrival of data. If any data are sensed during the extended on duration, UE stays in active mode. If no data are sensed, before and until the expiry of an inactivity timer, UE switches to a sleep mode by initiating the short DRX cycle $T_{s}$. The $T_{s}$ duration is a combination of $t_{o}$ and power off duration. UE wakes up for $t_{o}$ duration during $T_{s}$ while in sleep mode to detect any arrival of data in PDCCH channel. If data are sensed during this period, UE enters back to an active mode else it maintains the sleep mode. This detection and sleeping process sequence repeats for the duration of DRX short timer $N_{s}$ value. On expiry of $N_{s}$, the UE switches further to a deep sleep period of long DRX cycle $T_{l}$. However, the LTE DRX retains the UE in deep sleep mode till the next arrivals of data packets are sensed. This ensures the maximum power saving in the battery of UE. 


\section{Proposed QoS Support Technique}

In general, some of the arrived packets at the UE get discarded while contemporary arrival of packets occurs during the start of DRX mechanism. This deteriorates the enhanced QoS support of the LTE system. This major problem is addressed in the existing work ${ }^{14}$ with packet loss ratio analysis due to the delay bound violation. In the existing technique a regenerative cycle method has been adopted to address the packet loss ratio problem. However, the analyses for reducing the packet loss ratio in the work has not oriented to a specific traffic pattern. Moreover, the criterion for the selection of DRX parameters has not yet defined to balance the QoS and the power tradeoff.

In this paper, a new technique has been proposed to mitigate the packet loss ratio with optimum power savings for the delay bound violated traffic in the Telecommunication Standards Institute (ETSI) traffic model. The selection of DRX parameter namely On Timer duration to the ratio of Short DRX Cycle (OTSC) has been proposed and incorporated in the analysis as index $\alpha$. The selection $\alpha$ index has been identified to be below 1. Incorporation of $\alpha$ with the value of land above increases the power consumption. Hence, for the analysis $\alpha$ is selected as $0<\alpha<1$ to maintain the QoS support for reducing the packet loss ratio in the delay bound violated traffic. Impact of $\alpha$ has also been analyzed with the packet buffer P in order to maintain the QoS in the LTE network for GBR transmission.

The next section illustrates the quantitative method of analysis to reduce the packet loss ratio by incorporating the index value $\alpha$.

\section{Analysis on Packet Loss Ratio}

The packet loss ratio is analyzed based on the packets handled during different states of UE. It has been assumed that the packets sensed during these states are the busy period $\mathrm{B}$, contemporary arrival period $\mathrm{C}$ and the sleep period S. The value of OTSC ratio $\alpha$, is selected with the value between 0,1 and $\mathrm{L}$ is assumed to be the number of packets lost.

As the activity of UE has been divided in three parts, the energy consumed during the busy period (B) contemporary arrival period $(\mathrm{C})$ and sleep period $(\mathrm{S})$ are given as,

$$
\begin{gathered}
P_{B}=\frac{1-e^{-\lambda t_{i}}}{e^{-\lambda t_{i}}(1-\lambda)} \\
P_{c}=\frac{\lambda}{1-\lambda}\left[\left(1-e^{-\lambda \frac{t_{o} N_{s}}{\alpha}}\right)\left(\Omega+\frac{(1-\Omega) \lambda \frac{t_{o}}{\alpha}}{1-e^{-\lambda \frac{t_{o}}{\alpha}}}\right)+e^{-\lambda \frac{T_{o n} N_{s}}{\alpha}}\left(\Omega+\frac{(1-\Omega) \lambda T_{T}}{\left(1-e^{-\lambda T_{i}}\right)}\right)\right]
\end{gathered}
$$

For calculating the energy consumed during sleep period $S$,

Let us assume that

$$
e^{-\lambda}+\lambda e^{-\lambda} \cong 1
$$

However, when considering the packets due to the violation of delay bound, it can be dropped and moreover by neglecting the possibilities during the sleeping state, it is stated to be equivalent to $C$. Thus the energy during the sleep is given as

$$
P_{S}=P_{C}
$$

The summation of equations (1), (2) and (3) provides the total energy PT consumed by the UE during the three periods and it is given as

$$
P_{T}=P_{B}+P_{C}+P_{S}
$$

The energy consumed by UE during the number of packets lost is given as

$$
\begin{gathered}
P_{N}=S-\left[\left(1-e^{-\lambda \frac{t_{o} N_{s}}{\alpha}}\right)\left(\Omega+\frac{(1-\Omega) \lambda \frac{t_{o}}{\alpha}}{1-e^{-\lambda \frac{t_{o}}{\alpha}}}\right)+e^{-\lambda \frac{T_{o s} N_{s}}{\alpha}}\left(\Omega+\frac{(1-\Omega) \lambda T_{l}}{\left(1-e^{-\lambda T_{l}}\right)}\right)\right] \\
\text { where } \Omega=1-e^{-\lambda T_{o n}^{\prime}} \text { and } T_{o n}^{\prime}=t_{o}-1 \\
S=t_{s}+t_{l}
\end{gathered}
$$

Therefore, the Equations 4 and 5 becomes the governing ratio of the packet loss ratio $\mathrm{Pl}$ and it is given as

$$
P_{l}=\frac{P_{N}}{P_{T}}
$$

\section{Simulation Results and Discussion}

The packet loss ratio analyses with different DRX parameters are done using the MATLAB simulator. Table 2 shows the parameters used in simulation that are specified in the standard LTE DRX parameter range values. The packet loss ratio analyses of the proposed model illustrated from the Figures 2 to 6 are compared with the existing work ${ }^{14}$. 
Table 2. Simulation parameters

\begin{tabular}{|l|c|}
\hline DRX Parameter & Standard Value \\
\hline$\alpha$ & $0.1,0.0625$ \\
\hline$\lambda$ & 0.01 \\
\hline Inactivity Timer $t_{i}$ & $1 \mathrm{~ms}$ \\
\hline Long DRX cycle $T_{l}$ & $256 \mathrm{~ms}$ \\
\hline Packet Buffer P & $150 \mathrm{~ms}$ \\
\hline Short Cycle Timer $N_{s}$ & 8 \\
\hline
\end{tabular}

Figure 2 illustrates the performance analysis on packet loss ratio verses DRX short cycle timer. With a constant packet arrival rate of $\lambda=0.01$, the packet loss ratio is reduced when the DRX short cycle timer is increased. Moreover, the impact of $\alpha$ shows that a further reduction in the packet loss ratio is obtained by selecting a smaller value of $\alpha$. The figure depicts that by reducing the DRX short cycle timer, the packet loss ratio increases as a reason of lesser involvement of on timer duration during DRX sleep mode. The reduction in $63.6 \%$ of packet loss ratio is achieved at $\alpha=0.0625$ for $N_{s}=8$.

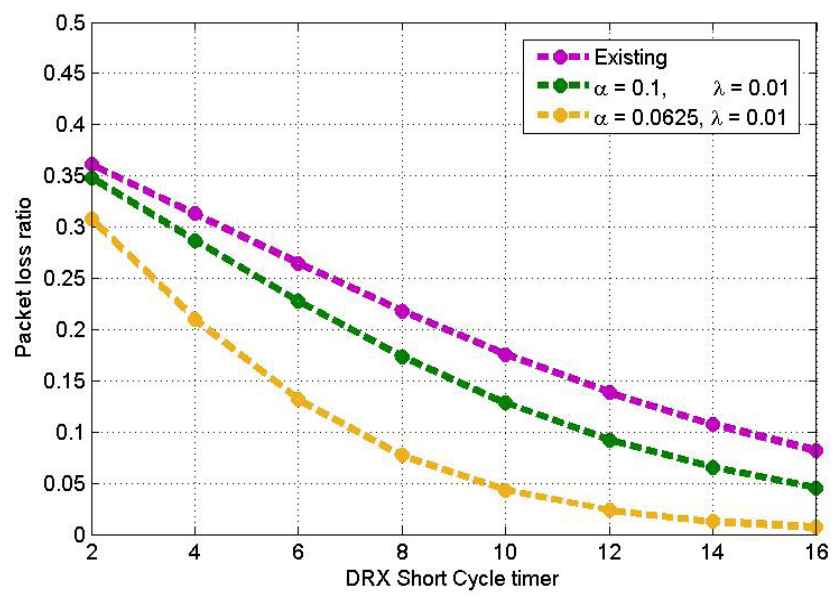

Figure 2. Packet loss ratio vs DRX short cycle timer.

The power savings in the battery depends on the switch off duration of UE. Hence, it is influenced by the long DRX cycle parameter. Figure 3 depicts the influence on the packet loss ratio obtained by increasing the long DRX cycle value. The loss ratio increases when long DRX cycle is increased which proves that the packets are getting discarded due to the longer durations between the subsequent on timer period. However, the selection of $\alpha=0.0625$ shows that the packet loss ratio reduces to $9.33 \%$ when $T_{l}$ is selected between the ranges $320 \mathrm{~ms}$ to $1024 \mathrm{~ms}$.

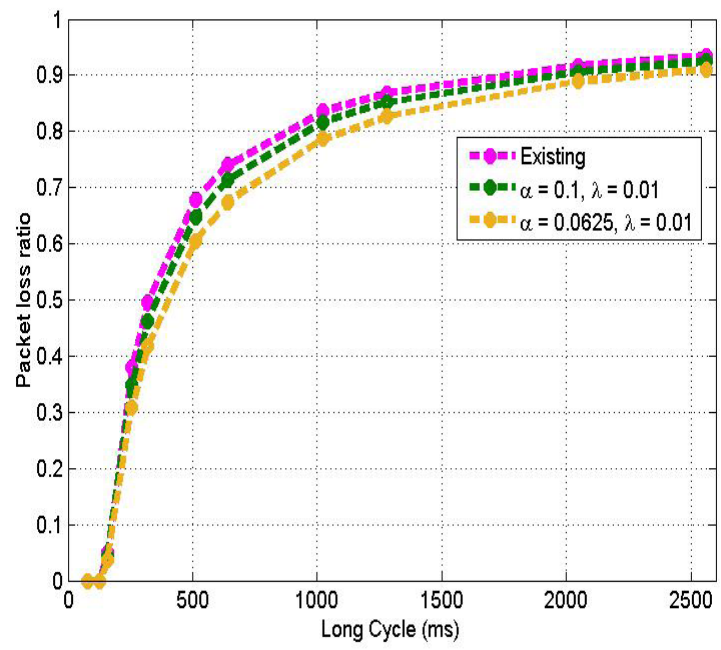

Figure 3. Packet loss ratio vs long drx cycle.

Figure 4 shows the effects of inactivity timer on the performance of packet loss ratio. Higher values of inactivity timer provides more duration for UE to sustain in active mode. Hence the chances of packet discarding are reduced. The figure also shows that lower inactivity timer increases the packet loss ratio as the duration of active period decreases. The figure also reveals that $25 \%$ of improvement in the reduction of packet loss ratio is achieved when $\alpha=0.0625$ is maintained.

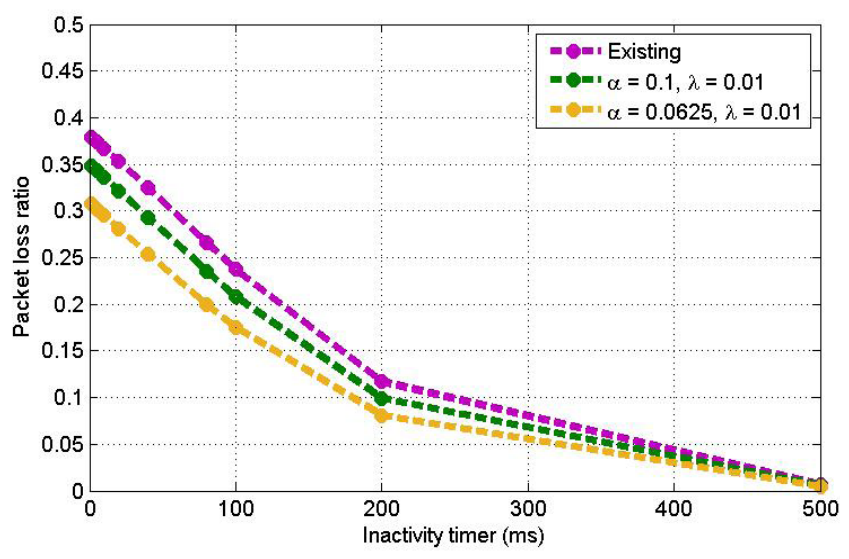

Figure 4. Packet loss ratio $v s$ inactivity timer.

Another performance study on packet loss ratio is made with different packet arrival rate. Figure 5 represents the impact of packet loss ratio for various values of packet mean arrival rate. In general, higher the packet arrival rate keeps the UE in active mode for longer duration. More active period of UE which in turn reduces the chance of packets discarding during the delay bound 
violated traffic. With $T_{l}=256 \mathrm{~ms}$ and buffer packet $P=$ 150 , the figure also illustrates an improvement in reduction of packet loss ratio when the mean packet arrival is increased. The selection of $\alpha$ value in addition to it shows that $47.05 \%$ of improvement in the reduction of packet loss ratio is achieved.

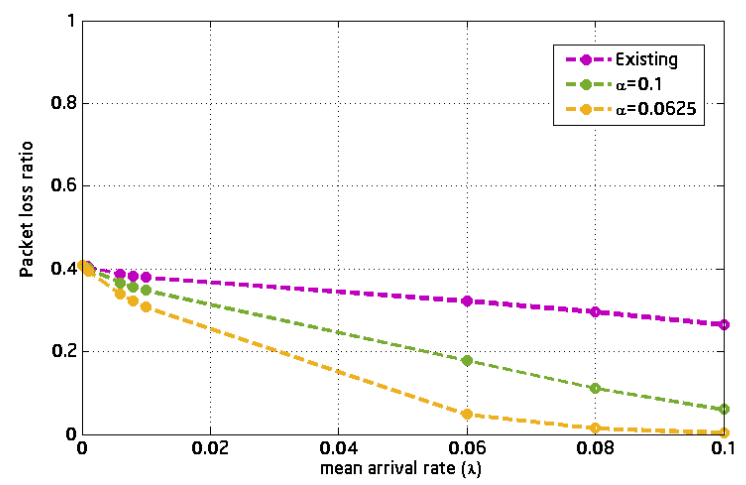

Figure 5. Packet loss ratio vs mean packet arrival rate.

The packet loss ratio analysis is performed for $\lambda=0.01$, $t_{i}=1 \mathrm{~ms}$ and $T_{l}=256 \mathrm{~ms}$ by varying the buffer size.Figure 6 depicts the response of the packet loss ratio for various values of buffer size. The figure also represents the reduction in the packet loss ratio when the buffer size increases. In general the LTE network has to maintain a Guaranteed Bit Rate (GBR) $150 \mathrm{~ms}$ of buffer size to provide the QoS for any video data service. Moreover, the figure shows that on reducing the buffer size below $150 \mathrm{~ms}$ increases the chance of packet loss ratio which deteriorates the QoS. Selection of buffer size below $150 \mathrm{~ms}$ increases the packet loss ratio and above $150 \mathrm{~ms}$ reduces packet loss ratio which in turn increases power consumption. Hence, it is inferred from the figure 6 that selection of buffer size decides to optimize the tradeoff between packet loss ratio and power savings.

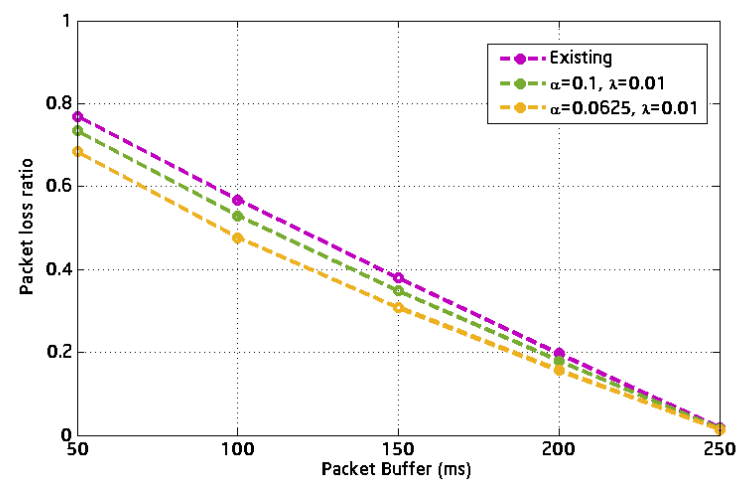

Figure 6. Packet loss ratio vs packet buffer.

\section{Conclusion}

In this paper a quantitative analysis on the the packet loss ratio is done by appending the OTSC ratio technique in DRX mechanism of LTE. The quantitative analyses are made with the MATLAB simulation. It is inferred through the simulation result that an improvement of $25 \%$ in the reduction of packet loss at $\alpha=0.0625$ on configuring the inactivity timer $T_{i}$ below $10 \mathrm{~ms}$. Packet loss reduction of $63.6 \%$ is achieved when DRX short cycle timer of Ns $=8$. Further a reduction of packet loss ratio of $9.33 \%$ is achieved when configuring long DRX cycle $T_{l}$ between $320 \mathrm{~ms}$ to $1024 \mathrm{~ms}$. Furthermore, configuration of buffer size to $150 \mathrm{~ms}$ shows an improvement of $15.78 \%$ in the reduction of packet loss ratio. It is also inferred from the analysis that selection of $\alpha$ below the index value 1 outperforms in reducing the packet loss ratio than that of existing work. It is concluded from the quantitative analysis that incorporation of OTSC in DRX mechanism with lower value of $\alpha$ and the optimal value of the buffer size selection provides better solution to enhance the QoS requirement in the LTE.

\section{References}

1. Varma SR, Krishna M, Sivalingam S, Tung L, Lin Y. Analytical Model for Power Savings in LTE Networks using DRX Mechanism. Mumbai, India: IEEE 21st National Conference on Communications, 2015 Mar. p. 1-6.

2. 3GPP, Requirements for Evolved UTRA (E-UTRA) and Evolved UTRAN (E-UTRAN). 2013. Available from: http:// www.3gpp.org

3. ETSI Technical Report UMTS 30.03 VERSION 3.2.0: Universal Mobile Telecommunications System (UMTS): Selection Procedures for the Choice of Radio Transmission Technologies of the UMTS. Data accessed: 1998. Available from: www.etsi. org/deliver/etsi_tr/101100.../03.../tr_101112v030200p.pdf.

4. Yang SR, Lin SB. Modeling UMTS Discontinuous Reception Mechanism. IEEE Transaction on Wireless Communications. 2005 Jan; 4(1):312-19.

5. Yang SR, Yan S, Hung HN. Modeling UMTS Power Saving with Bursty Packet Data Traffic. IEEE Transaction on Mobile Computing. 2007; 6(12):1398-409.

6. Kolding TE, Wigard J, Dalsgaard L. Balancing power saving and single user experience with discontinuous reception in LTE. Siena-Tuscany, Italy: Proceeding of IEEE International Symposium on Wireless Communication Systems (ISWCS’09); 2009 Sept. p. 713-17. 
7. Huang J, Qian F, Gerber A, Mao ZM, Sen SO. Spatscheck. A Close Examination of Performance and Power Characteristics of 4G LTE Networks. Ambleside UK: Proceeding of ACM; 2012. p. 225-38.

8. Ali T, Satish KC, Rath J, Vannithamby V, Torlak M. Device Power Saving and Latency Optimization in LTE-A Networks through DRX Configuration. IEEE Transaction on Wireless Communications. 2014; 13(5):2614-25.

9. Zhou L, Xu H, Tian H, Gao Y, Du L, Chen L. Performance Analysis of Power Saving Mechanism with Adjustable DRX Cycles in 3GPP LTE. Calgary, BC: Proceeding of IEEE 68th Vehicular Technology Conference; 2008. p. 1-5.

10. Kim S, Jung K, Choii J, Kwak Y. Improving LTE/LTE-A UE Power Efficiency with Extended DRX Cycle. Vancouvaer, BC: Proceeding of IEEE 80th Vehicular Technology Conference; 2014. p. 1-5.

11. Yang CC, Mai YT, Chen JY, Kuo YC. LBPS: Load Based Power Saving in the IEEE 802.16e Network. Computers and Electrical Engineering. 2012 Jul; 38(4):91-95.
12. Yang CC, Mai YT, Chen JY, Kuo YC. Integrated Load Based Power Saving for BS and MSS. IEEE 802.16e Network. Wireless Communications and Mobile Computing. 2015 Mar; 15(4):601-14.

13. Yang CC, Chen JY, Mai YT, Liang CH. Design of a Load Based DRX Scheme for Non-Real-Time Traffic in LTE. Hong Kong: Proceeding of the International Multi-Conference of Engineers and Computer Scientists; 2014 Sep. p. 197-200.

14. Lee TH, Tsai CH, Wu TH. Quality of Service Support under DRX Mechanism in LTE Advanced Wireless Networks. Dresden: Proceeding of the IEEE 77th Vehicular Technology Conference; 2013 Jun. p. 1-5.

15. Radhakrishnan S, Neduncheliyan S, Thyagharajan KK. A review of downlink packet scheduling algorithms for real time traffic in LTE-Advanced Networks. Indian Journal of Science and technology. 2016 Jan; 9(4):1-4. 